\title{
Status of ToRCH positivity among the children presented with congenital Hydrocephalus
}

\author{
Mukherjee $\mathrm{SK}^{1}$, Afreen $\mathrm{S}^{2}$, Roy $\mathrm{HA}^{3}$, Khan $\mathrm{RK}^{4}$, Arman DM ${ }^{5}$, Hossain $\mathrm{MA}^{6}$, Ziauddin ${ }^{7}$, \\ Ekramullah $\mathrm{SM}^{8}$, Rahman $\mathbf{M}^{9}$, Yousuf $\mathrm{A}^{10}$, Islam $\mathrm{J}^{11}$
}

Conflict of Interest: None

Funding: No funding agency. How to Cite This Article:

Contribution to authors: SKM is the principle investigator, S.A, M.R, D.M.A, J.I, S.M.E associated in data collection. H.R, Z.H, M.A.H, help for editorial formatting. M.A.Y helps for serological testing.

Copyright: @2020bang.BJNS published by BSNS. This article is published under the creative commons CC-BY-NC license. This license permits use distribution (https://creativecommons orgf/licences/by-nc/4-0/)reproduction in any medium, provided the original work is properly cited and is not used for commercial purposes.

Received: 4 June 2019

Accepted: 16 April 2019

\begin{abstract}
: secondary to Tumor or Trauma was excluded from this study. Toxoplasma.

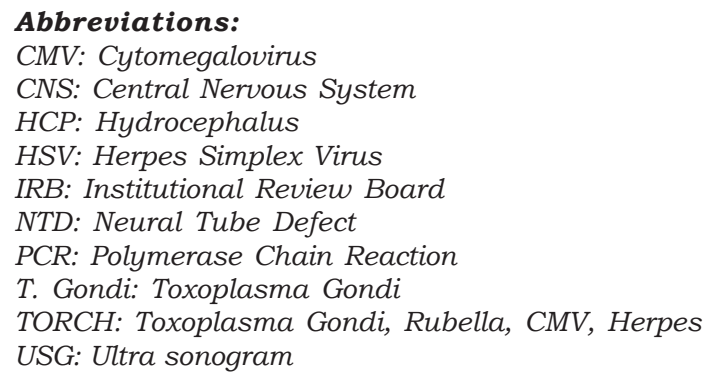

Introduction: ToRCH is an acronym for four congenital infections that are sometimes difficult to distinguish: Toxoplasmosis, Rubella, Cytomegalovirus (CMV), and Herpes Simplex Virus (HSV). This group of organisms produces serious Central Nervous System (CNS) and other infections, which are potentially preventable and treatable.

Methods: Neonates and infants with hydrocephalus $(\mathrm{N}=65)$ identified via the outpatient department of the National Institute of Neurosciences and Hospital (NINS\&H), were prospectively screened for ToRCH antibodies. Hydrocephalus

Results: Evidence of $75.38 \%(p=<0.05)$ patient was positive for TORCH antibodies. Toxoplasma Gondi (T. Gondii) IgG $18.5 \%$ ( $p=<0.05$ ), CMV IgM $9.2 \%(p=<0.05)$, CMV Ig G47.7\% ( $p=<0.5)$, Rubella, and HSV $1 \& 2$ antibodies were also identified.

Conclusion: The vast majority of cases of neonatal hydrocephalus at one institution was post-infectious in etiology and related to ToRCH infections. Many of these cases could be prevented with improved screening and treatment. While study of a single institution may not be the representative of the entire population, further study and implementation of a standardized screening protocol would likely benefit this population.

Key Words: Congenital Infection, ToRCH, Hydrocephalus, Prevention, CMV,

Bang. J Neurosurgery 2020; 9(2): 121-125

1. Dr. Sudipta Kumer Mukherjee, Assistant Professor, Department of Pediatric Neurosurgery, National Institute of Neurosciences \& Hospital, Sher-E-Bangla Nagar, Dhaka-1207

2. Dr. D. M. Arman, Assistant Professor, Department of Clinical Neurosurgery, National Institute of Neurosciences \& Hospital, SherE-Bangla Nagar, Dhaka-1207

3. Dr. Holly A Roy, Academic Clinical Fellow in Neurosurgery Department, Derriford Hospital, Plymouth, PL6 8DH (UK)

4. Dr. Rafiul Karim Khan, Assistant Professor, Department of Neurosurgery, Shaheed Suhrawardy Medical College Hospital

5. Dr. Samantha Afreen, Major, Classified Specialist, Neurosurgery, $\mathrm{CMH}$

6. Dr. Muhammad Arif Hossain, Medical Officer, Department of Pediatric Neurosurgery, National Institute of Neurosciences \& Hospital, Sher-E-Bangla Nagar, Dhaka-1207

7. Dr. Md. Ziauddin, Assistant Registrar, Department of Pediatric Neurosurgery, National Institute of Neurosciences \& Hospital, SherE-Bangla Nagar, Dhaka-1207

8. Prof. Sk. Md. Ekramullah, Professor \& Head, Associate Professor, Department of Pediatric Neurosurgery, National Institute of Neurosciences \& Hospital, Sher-E-Bangla Nagar, Dhaka-1207

9. Dr. Md. Moshiur Rahman, Medical Officer, Department of Pediatric Neurosurgery, National Institute of Neurosciences \& Hospital, Sher-E-Bangla Nagar, Dhaka-1207

10. Dr. Md. Abdullah Yusuf, Assistant Professor, Department of Microbiology, National Institute of Neurosciences \& Hospital, Sher-EBangla Nagar, Dhaka-1207

11. Dr. Md. Joynul Islam, Associate Professor, Department of Clinical Neurosurgery, National Institute of Neurosciences \& Hospital, Sher-E-Bangla Nagar, Dhaka-1207

Address of Correspondence: Dr. Sudipta Kumer Mukherjee, Associate Professor, Department of Pediatric Neurosurgery, National Institute of Neurosciences \& Hospital, Sher-E-Bangla Nagar, Dhaka-1207 email: sudipta70@hotmail.com, Cell No: +880-1711709096 


\section{Manuscript:}

Introduction: ToRCH infections cause significant morbidity and mortality in neonates. Transplacental spread is the usual route of infection in neonates. Post-infectious hydrocephalus caused by congenital ToRCH infections is a significant source of morbidity and mortality, and its prevalence is known to vary by region. This pilot study was done to assess the association between Congenital HCP and $\mathrm{ToRCH}$ infection. Early diagnosis and treatment of congenital ToRCH infections leads to improved outcomes. In an Indian study in Hyderabad 28\% T. Gondii was present and CMV was present in $92 \%$ population among pregnant woman. ${ }^{1}$ Post-infectious etiology has been shown to be more prevalent in low - and middle - income countries compared with high - income countries. Congenital toxoplasmosis may lead to a wide range of ocular and neurologic sequel, including hydrocephalus, although avoiding exposure to cats and uncooked meat can aid prevention. Toxoplasma tachyzoites are secreted into milk in various farm animals. ${ }^{2}$ Hydrocephalus has been estimated to affect approximately $4 \%$ of infants with congenital toxoplasmosis and is a signiûcant cause of morbidity and mortality in this disease. ${ }^{3}$ The incidence of congenital rubella syndrome was significantly reduced over the last century due to vaccination. ${ }^{4} \mathrm{CMV}$ is the most common cause (10$20 \%$ ) of congenital infection in the United States. ${ }^{5,6}$ Such infants have a mortality rate of $12 \%$ by six months of age. ${ }^{7}$ Global prevalence of CMV infection in developed countries (45\%) and developing countries $(100 \%) .{ }^{8}$ Herpes simplex is most often acquired during delivery rather than during gestation, it is more preventable and treatable compared with CMV or Rubella. $^{9}$

\section{Material and Method:}

This was a prospective observational study, was conducted in Pediatric Neurosurgery department of National Institute of Neurosciences and Hospital (NINS\&H). Dhaka, Bangladesh. Sixty five (65) patients were recruited from November 2017 to October 2018. Inclusion criteria were neonatal HCP age $<1$ year. Exclusion criteria were HCP associated with tumor, trauma, hemorrhage, arachnoid cyst, and Dandy Walker Malformation. As per inclusion \& exclusion criteria we recruit sixty five (65) cases and do serological examination [ELISA, ERBA ${ }^{\circledR}$, USA $^{10}$ ]. A literature review regarding $\mathrm{ToRCH}$ related $\mathrm{HCP}$, including screening and treatment protocols was also performed. Data were analyzed for association between Neonatal HCP and Congenital ToRCH infection using SPSS17. Categorical Data we analyzed using one sample t test where $p$ Value $<0.05$ was considered statistically significant. This study was approved by Institutional Review Board (IRB).

\section{Results:}

A total 65 cases of Congenital HCP recruited as per selection criteria. Among the 65 cases $75.38 \%$ cases were ToRCH Positive (Fig.1). Most of the patients came from rural area, $56.9 \%$ were male.

Negative Positive

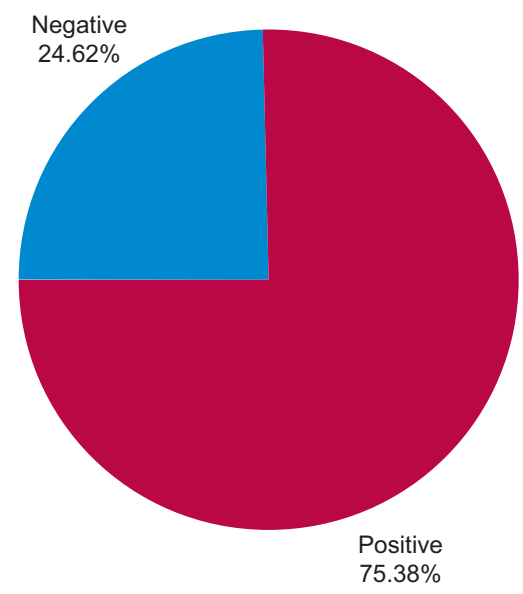

Fig.-1: Pie Chart of ToRCH positive patient.

The commonest age group was $31-90$ days (41.5\%) (Fig. 2). The total age range was 26 days to 6 years. All three patients with concurrent encephalocele and all eight patients with concurrent myelomeningoceles were also found to have congenital ToRCH infections.

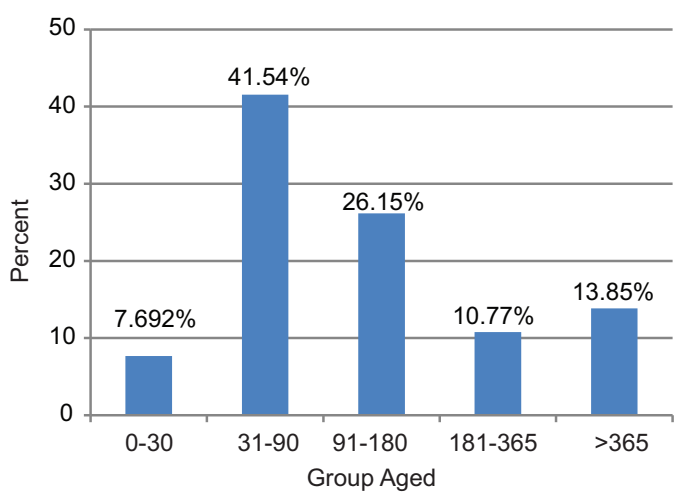

Fig .-2: Bar Chart for Age Distribution of ToRCH positive Patients. 


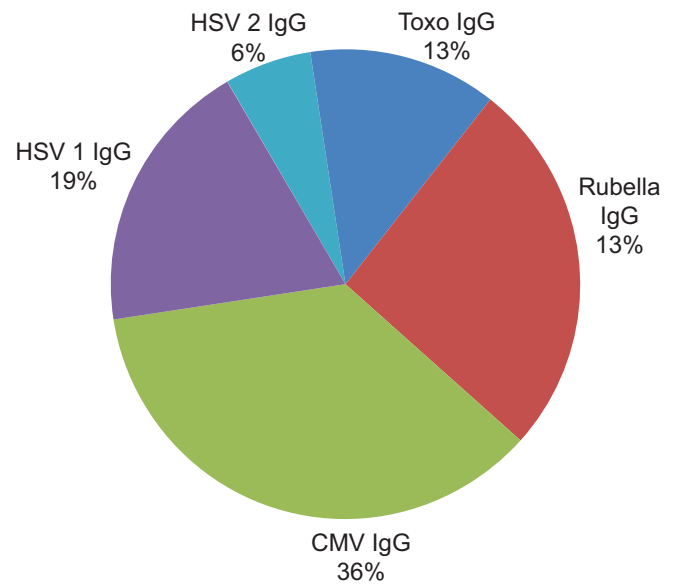

Fig.-3: IgG antibody among ToRCH positive patients

T. Gondii IgM antibody was absent but $18.5 \%$ were $T$. Gondii IgG positive $(p<0.05)$. Among the 12 cases of toxoplasma positive group $6(50 \%)$ had contact with cat during pregnancy. Rubella IgM \& IgG Antibody was present in $1.5 \%$ and $38.5 \%$ respectively. As $86 \%$ of population under coverage of vaccination, so, antibody against Rubella may not important. ${ }^{11}$ (Fig I.b)

Table-I

Distribution of T. Gondi IgG, CMV (IgM \& IgG), HSV (IgM \& IgG) Antibody.

\begin{tabular}{lcc}
\hline & Frequency & Percent \\
\hline T. Gondii IgG & 12 & 18.5 \\
CMV IgM & 6 & 9.2 \\
CMV IgG & 31 & 47.7 \\
HSV-1 IgM & 3 & 4.6 \\
HSV-1 IgG & 17 & 26.2 \\
HSV-2 IgG & 6 & 9.2 \\
\hline
\end{tabular}

CMV IgM \& IgG Antibody was present in $9.2 \%(p=<$ $0.05)$ and $47.7 \%(p=<0.05)$ respectively. HSV 1 $\lg M \& \lg G$ Antibody were present in $4.6 \%$ and $26.2 \%$ respectively. Herpes 2 IgM Antibody was absent but 9.2\% patients were HSV 2 IgG Positive.

\section{Discussion:}

During the past 20 years, a number of scientific and sociological changes have altered the scenario of ToRCH infections. The incidence of these diseases has changed significantly because there are better preventive measures. The potentially significant relation between ToRCH infections and CNS anomalies including HCP and also Neural Tube Defects (NTDs) is now an important subject of investigation. Few previous articles address this issue. Although maternal TORCH infection is common in Bangladesh, India, Malaysia and Thailand. ${ }^{12}$

The classic triad of $T$. Gondii inection is: Chorioretinitis, Hydrocephalus, Intracranial calcifications, and are present concurrently in $61.6 \%$ of cases. In 1982 Joksim et all did a study in Yugoslavia where they found $39 \%$ congenital HCP was T. Gondii positive, in our study $18.5 \%$ HCP was T. Gondii positive. ${ }^{13}$ In 2015 Samuel Huston's study demonstrated 4\% congenital HCP associated with congenital T. Gondii. ${ }^{14}$ Specific anatomical patterns of Toxoplasma infection commonly causes cerebral calciûcation and generalized periventricular inûammation (asymmetrical dilatation of the lateral ventricles ). ${ }^{15}$ Treatment for toxoplasmosis in utero and during the first year of life has been shown to significantly improve outcomes.

Rubella is a benign, self-limited viral illness. Since the availability of mass immunization, the reported incidence of rubella has dropped significantly. ${ }^{16}$ The prevention of congenital rubella is dependent upon adequate early immunization. Hydrocephalus may be due to leptomeningitis and is a very rare presentation in congenital rubella syndrome with very few cases been reported in literature. ${ }^{17}$

CMV is the most prevalent infectious agent causing neurological dysfunction in the developing brain. In addition to the transplacental route, CMV can be transmitted at delivery via the maternal genital tract, breast milk, and in transfused blood products. CMV is easily spread in daycare centers and in young children. Approximately $40 \%$ of maternal primary infections are transmitted to the fetus. However, first trimester primary maternal infection is more likely to cause neonatal infection that is evident at birth. Transmission of CMV from the mother to fetus can occur even if the mother was infected long before conception. A meta-analysis of fetal ultrasound findings indicates an association between CMV congenital infection and hydrocephalus in $4.7 \%$ of pregnancies. ${ }^{18} \mathrm{CNS}$ anomalies induced by congenital CMV infection are likely to result from a direct effect of viral replication in the brain and in the placenta. $\mathrm{HCP}$ in CMV as a result of ventriculitis and inflammatory infiltrate of $T$ lymphocytes in the 
ependyma, which can detected in antenatal Ultrasonogram (USG). ${ }^{19}$ In our study we found 9.2 $\%$ of HCP due to recent CMV infection and $47.7 \%$ $\mathrm{HCP}$ had IgG Antibody of CMV indicate maternal infection at least 3 months before. Congenital CMV infection is diagnosed by culturing the virus in oral secretions or urine, in the first three weeks of life. Transmission of the virus requires direct contact with body fluids. Thorough hand washing and other preventive hygienic measures can decrease spread in daycare centers and at home. ${ }^{20}$

It is interesting that neonatal infection occurs far less frequently than might be expected given the high prevalence (one in five) of seropositivity to HSV-2 in childbearing women. ${ }^{21}$ Known complications of intrauterine HSV infection include seizures, lethargy, irritability, tremors, poor feeding, temperature instability, bulging fontanelle, chorioretinitis, microcephaly, microphthalmia, and hydranencephaly. 22 In our Study HSV-1 IgM positive in 3\%, IgG positive in $26.2 \%$. HSV-2 IgM not found in any case. Whereas HSV-2 IgG present in $9.2 \%$. So, HSV-1 IgG present significantly $(P<.05)$. Literature review indicates relation between congenital HCP and Herpes infection is very weak.

Limitation: This study used serology as a diagnostic tool however Polymerase Chain Reaction (PCR) is more sensitive and specific. Other limitations were that the birth and recruitment interval was not fixed. History regarding immunization of baby, seropositivity of mother and contact with disease person was not recorded.

Proposal: Results of this study is a primary notice, as we face this profound problem we need to focus on this issue by further study. Then we can advice routine maternal and newborn screening as well as enhance preventive activity in high risk people.

\section{Conclusion:}

Congenital ToRCH infection and CNS involvement especially HCP is a severe problem for new born babies which produces serious socio-economic burden. This is an urgent issue to prevent, treat and do further research on this disease.

\section{References:}

1. K. Rebekah Prasoona, B. Srinadh, T. Sunitha, et al Seroprevalence and Influence of Torch Infections in High Risk Pregnant Women: A Large Study from South India. J Obstet Gynaecol India 2014; 65(5): 301-309: doi: 10.1007/ s13224-014-0615-3.
2. Helieh S. Oz, Maternal and congenital toxoplasmosis, currently available and novel therapies in horizon. Front Microbiol 2014; 5:385. Published 2014 Jul 24. doi:10.3389/ fmicb.2014.00385.

3. Swisher $\mathrm{CN}$, Boyer $\mathrm{K}$, McLeod $\mathrm{R}$ Congenital toxoplasmosis. Semin Pediatr Neurol 1994; 1(1): 4-25.

4. National Center for Immunization and Respiratory Diseases (NCIRD), Division of Viral Diseases. (2017). Retrieved from https://www.cdc.gov/rubella/about/in-theus.html

5. Harold Chen, Congenital Cytomegalovirus Infection. Atlas of Genetic Diagnosis and Counseling 2017; doi: 10.1007/ 978-1-4939-2401-1_50

6. Suresh B. Boppana, Karen B. Fowler, Robert F. Pass, et al Congenital Cytomegalovirus Infection: Association between Virus Burden in Infancy and Hearing Loss, Journal of Pediatrics, June 2005, 146(6): 817-823, DOI: https://doi.org/10.1016/j.jpeds.2005.01.059

7. Uduak S. Akpan, Pillarisetty LS, Congenital Cytomegalovirus Infection (Congenital CMV Infection). Treasure Island (FL): Stat Pearls 2019. https:// www.ncbi.nlm.nih.gov/books/NBK541003/

8. Landolfoa S, Garigliob M, Gribaudoa G, et al The human cytomegalovirus. Pharmacol Ther. 2003; 98(3):269-297. doi: 10.1016/S0163-7258(03)00034-2.

9. Sue G. Boyer, MN, RN, et al Newborn \& Infant Nursing Reviews, Update on TORCH Infections in the Newborn Infant. Newborn and Infant Nursing 2004;4(1)

10. Elabscience $囚$. https://www.elabscience.com/Productselisa_kits-61.html? gclid=CjwKCAjw4ZTqBRBZ EiwAHHxpftJMW TeVolqr SnZsod Cm7210XzSzImr NO_syPR4pO6moAAkkSzz2exoCqOwQAvD_BwE

11. Sheikh N , Sultana M , Ali N, Akram R, Mahumud RA, Asaduzzaman ${ }^{\mathrm{M}^{*}}$ et al. Coverage, Timelines, and Determinants of Incomplete Immunization in Bangladesh. Trop. Med. Infect. Dis. 2018, 3(3): 72; https://doi.org/ 10.3390/tropicalmed3030072

12. Nabi SN, Wasey AFCA, Haider KMTS et al Seroprevalence of TORCH antibody in Pregnant Woman. JAFMC, Bangladesh, 2012; 8,(1): 35-39: DOI: http://dx.doi.org/ 10.3329/jafmc.v8i1.13537.

13. Joksim M., Desanka Š V.D., Mom ilo D., et al. Frequency of toxoplasmosis in the appearance of congenital hydrocephalus. J Neurosurgery. 1982; 56(6):830-4. doi: https://doi.org/10.3171/jns.1982.56.6.0830.

14. Hutson. S. L., Wheeler. K. M., McLone D., et al Patterns of Hydrocephalus Caused by Congenital Toxoplasma gondii Infection Associate with Parasite Genetics. Clinical Infectious Diseases, 2015;61,(12): 18311834, doi:10.1093/cid/civ720.

15. Simeone RM, Rasmussen SA, Mei JV, et al: A pilot study using residual newborn dried blood spots to assess the potential role of cytomegalovirus and Toxoplasma gondii in the etiology of congenital hydrocephalus. Birth Defects 
Res A Clin Mol Teratol 2013;97(7): 431-436, doi: 10.1002/ bdra.23138.

16. Berger BE, Navar-Boggan AM, Omer SB. Congenital rubella syndrome and autism spectrum disorder prevented by rubella vaccination-United States, 2001-2010. BMC Public Health. 2011;11: 340. 2011 May 19. doi:10.1186/14712458-11-340

17. Tiwari C, Sandlas G, Jayaswal S, Shah H. Hydrocephalus in Congenital Rubella Syndrome: A Case Report. J Immunol Infect Dis. 2014. 2(1): 102. doi: 10.15744//2394-6512.1.202

18. Benoist $\mathrm{G}$, Ville $\mathrm{Y}$ : Fetal infection, in Rodeck $\mathrm{CH}$, Whittle MJ (eds): Fetal Medicine: Basic Science and Clinical Practice, ed 2. New York: Churchill Livingstone Elsevier, 2009, pp 620-641.
19. Gabrielli. L., Bonasoni. M.P., Santini.D. et. al. Congenital cytomegalovirus infection: patterns of fetal brain damage. Clin Microbiol Infect 2012. 18(10) 419-427: doi: https:// doi.org/10.1111/j.1469-0691.2012.03983.x

20. Nesti MM, Goldbaum M. Infectious diseases and daycare and preschool education. J. Pediatr. (Rio J.) 2007; 83(4): 299-312. doi.org/10.2223/JPED.1648.

21. Kimberlin DW, Neonatal herpes simplex infection. Clin Microbiol Rev. 2004; 17(1):1-13. doi:10.1128/cmr.17.1.113.2004

22. Sloan JK, Cawyer CR, Drever NS. Fetal ventriculomegaly and herpes encephalitis following primary maternal herpes simplex infection. Proc (Bayl Univ Med Cent). 2017;30(4):463-464. doi:10.1080/08998280. 2017. 11930231. 